\title{
THE CURIOUS ADVENTURE OF THE ULTRAHIGH ENERGY COSMIC RAYS
}

\author{
F. W. Stecker \\ Laboratory for High Energy Astrophysics, Code 661, NASA/Goddard Space Flight Center, \\ Greenbelt, MD 20771, USA.
}

\begin{abstract}
I will discuss the mysteries involving the production and extragalactic propagation of ultrahigh energy cosmic rays and suggested possible solutions.
\end{abstract}

\section{INTRODUCTION}

About once per century per $\mathrm{km}^{2}$ of the Earth's surface, a giant shower of charged particles produced by a primary particle with an energy greater than or equal to 6 joules (100 $\mathrm{EeV}=10^{20} \mathrm{eV}$ ) plows through the Earth's atmosphere. The showers which they produce can be detected by arrays of scintillators on the ground; they also announce their presence by producing a trail of ultraviolet flourescent light, exciting the nitrogen atoms in the atmosphere. The existence of such showers has been known for almost four decades (Linsley 1963). The number of giant air showers detected from primaries of energy greater than 100 $\mathrm{EeV}$ has grown into the double digits and can be expected to grow into the hundreds as new detectors such as the "Auger" array and the "EUSO" (Extreme Universe Space Observatory) and "OWL" (Orbiting Wide-Angle Light Collectors) satellite detectors come on line. These phenomena present an intriguing mystery from two points of view: (1) How are particles produced with such astounding energies, eight orders of magnitude higher than 
are produced by the best man-made terrestrial accelerators? (2) Since they are most likely extragalactic in origin, how do they reach us from extragalactic distances without exhibiting the predicted cutoff from interactions with the $2.7 \mathrm{~K}$ cosmic background radiation? In these lectures, I will consider possible solutions to this double mystery.

\section{THE EVIDENCE}

Figure 1 shows the data on the ultrahigh energy cosmic ray spectrum from the Fly's Eye and Akeno detectors. Other data from Havera Park and Yakutsk may be found in the review by Nagano and Watson (2000) are are consistent with Figure 1. Additional data are now being obtained by the HiRes detector array and should be available in the near future (Abu-Zayyad, private communication).

For air showers produced by primaries of energies in the 1 to $3 \mathrm{EeV}$ range, Hayashida, et al. (1999) have found a marked directional anisotropy with a $4.5 \sigma$ excess from the galactic center region, a $3.9 \sigma$ excess from the Cygnus region of the galaxy, and a $4.0 \sigma$ deficit from the galactic anticenter region. This is strong evidence that EeV cosmic rays are of galactic origin.

As shown in Figure 2, at EeV energies, the primary particles appear to have a mixed or heavy origin, trending toward a protonic origin in the higher energy range around 30 EeV (Bird, et al. 1993; Abu-Zayyad, et al. 2000). This trend, together with evidence of a flattening in the cosmic ray spectrum on the 3 to 10 EeV energy range (Bird, et al. 1994; Takeda et al. 1998) give evidence for a new component of cosmic rays dominating above 10 EeV energy.

The apparent isotropy (no galactic-plane enhancement) of cosmic rays above $10 \mathrm{EeV}$ (e.g. Takeda, et al. 1999), together with the difficulty of confining protons in the galaxy at 10 to $30 \mathrm{EeV}$ energies, provide significant reasons to believe that the cosmic-ray component above $10 \mathrm{EeV}$ is extragalactic in origin. As can be seen from Figure 1, this extragalactic component appears to extend to an energy of $300 \mathrm{EeV}$. Extention of this spectrum to higher 


\section{FIGURES}

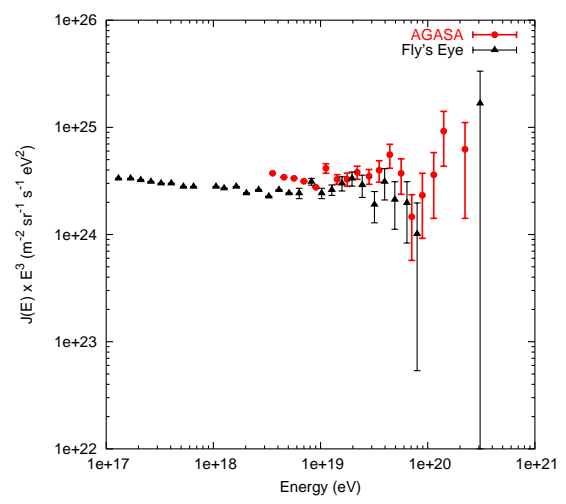

FIG. 1. The ultrahigh energy cosmic ray spectrum data from Fly's Eye and Akeno.

energies is conceivable because such cosmic rays, if they exist, would be too rare to have been seen with present detectors. We will see in the next section that the existence of 300 EeV cosmic rays gives us a new mystery to solve.

\section{THE GZK EFFECT}

Thirty five years ago, Penzias and Wilson (1965) reported the discovery of the cosmic $3 \mathrm{~K}$ thermal blackbody radiation which was produced very early on in the history of the universe and which led to the undisputed acceptance of the "big bang" theory of the origin of the universe. Much more recently, the Cosmic Background Explorer (COBE) satellite confirmed this discovery, showing that the cosmic background radiation (CBR) has the spectrum of the most perfect thermal blackbody known to man. COBE data also showed that this radiation (on angular scales $>7 \mathrm{deg}$ ) was isotropic to a part in $10^{5}$ (Mather et al. 1994). The perfect thermal character and smoothness of the CBR proved conclusively that this radiation is indeed cosmological and that, at the present time, it fills the entire universe with a $2.7 \mathrm{~K}$ spectrum of radio to far-infrared photons with a density of $\sim 400 \mathrm{~cm}^{-3}$. 


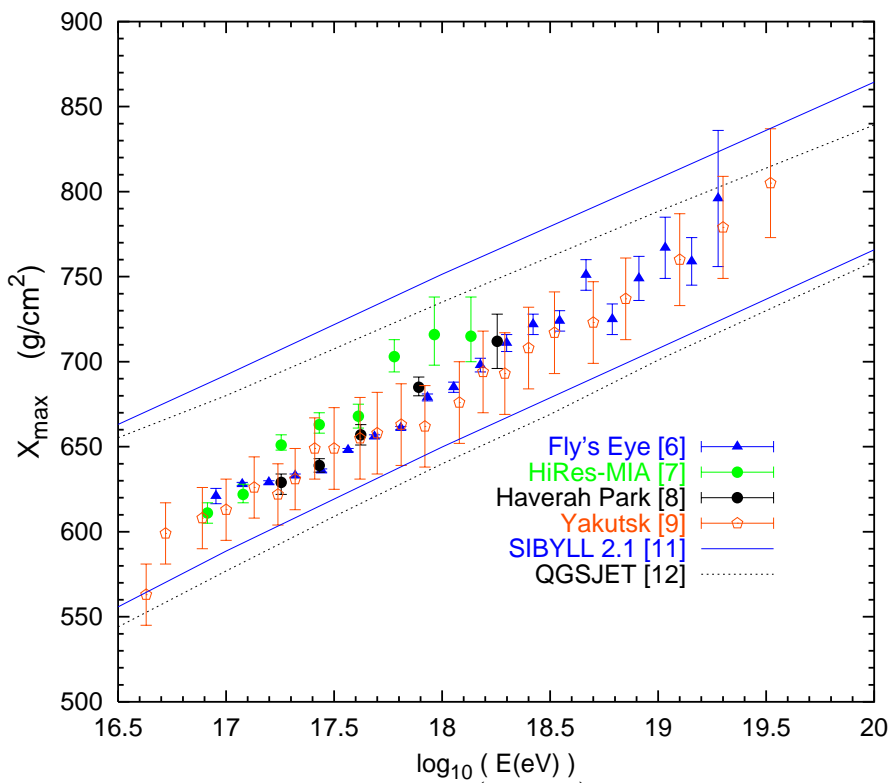

FIG. 2. Average depth of shower maximum $\left(X_{\max }\right)$ vs. energy compared to the calculated values for protons (upper curves) and Fe primaries (lower curves) (from Gaisser 2000; see references therein).

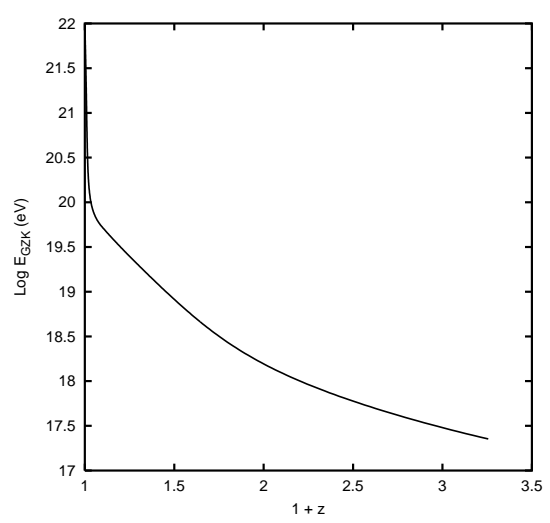

FIG. 3. The GZK cutoff energy versus redshift (Scully and Stecker 2000).

Shortly after the discovery of the CBR, Greisen (1966) and Zatsepin and Kuz'min (1966) predicted that pion-producing interactions of ultrahigh energy cosmic ray protons with CBR photons of target density $\sim 400 \mathrm{~cm}^{-3}$ should produce a cutoff in their spectrum at energies 
greater than $\sim 50 \mathrm{EeV}$. This predicted effect has since become known as the GZK (GreisenZatsepin-Kuz'min) effect. Shortly after the GZK papers, Stecker (1968) utilized data on the energy dependence of the photomeson production cross sections and inelasticities to calculate the mean energy loss time for protons propagating through the CBR in intergalactic space as a function of energy. Based on his results, Stecker (1968) then suggested that the particles of energy above the GZK cutoff energy (hereafter referred to as trans-GZK particles) must be coming from within the "Local Supercluster" of which we are a part and which is centered on the Virgo Cluster of galaxies. Thus, the "GZK cutoff" is not a true cutoff, but a supression of the ultrahigh energy cosmic ray flux owing to a limitation of the propagation distance to a few tens of Mpc.

The actual position of the GZK cutoff can differ from the $50 \mathrm{EeV}$ predicted by Greisen. In fact, there could actually be an enhancement at or near this energy owing to a "pileup" of cosmic rays starting out at higher energies and crowding up in energy space at or below the predicted cutoff energy (Puget Stecker and Bredkamp 1976; Hill and Schramm 1985; Berezinsky and Grigor'eva 1988; Stecker 1989; Stecker and Salamon 1999). The existence and intensity of this predicted pileup depends critially on the flatness and extent of the source spectrum, (i.e., the number of cosmic rays starting out at higher energies), but if its existence is confirmed in the future by more sensitive detectors, it would be evidence for the GZK effect.

Scully and Stecker (2000) have determined the GZK energy, defined as the energy for a flux decrease of $1 / e$, as a function of redshift. At high redshifts, the target photon density increases by $(1+z)^{3}$ and both the photon and initial cosmic ray energies increase by $(1+z)$. The results obtained by Scully and Stecker are shown in Figure 3. 


\section{THE DOG IN THE NIGHT TIME}

The lack of the expected "GZK cutoff" in the spectrum of ultrahigh energy cosmic rays is a case of the dog that did nothing in the night timef It is an important clue, possibly eliminating some suggested astrophysical sources for these cosmic rays and possibly pointing the way to new high energy physics.

\section{THE HUNT FOR THE ZEVATRONS}

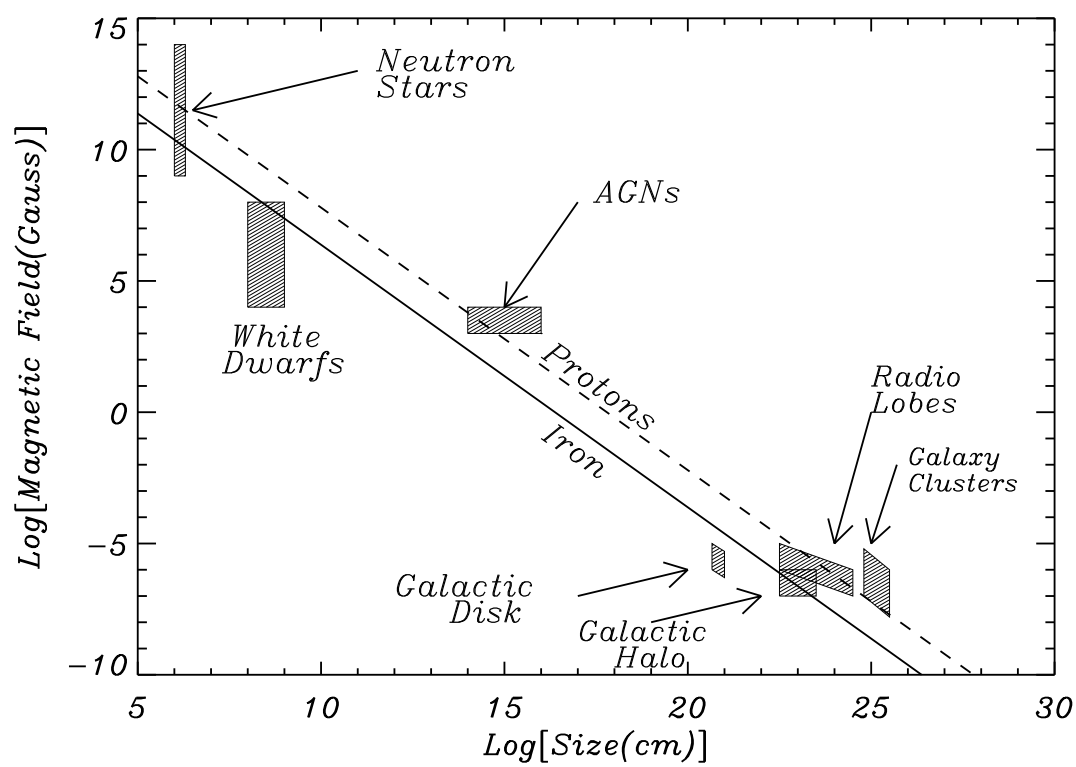

FIG. 4. A "Hillas Plot" showing potential astrophysical zevatrons (from Olinto (2000).

The lack of a GZK cutoff has led theorists to go on a hunt for nearby "zevatrons", i.e., astrophysical sources which can accelerate particles to energies $\mathcal{O}\left(1 \mathrm{ZeV}=10^{21} \mathrm{eV}\right)$.

In most theoretical work in cosmic ray astrophysics, it is generally assumed that the diffusive shock acceleration process is the most likely mechanism for accelerating particles

\footnotetext{
${ }^{1}$ In the Adventure of Silver Blaze by Arthur Conan Doyle, Sherlock Holmes noted the "curious incident of the dog in the night time". The absence of action on the part of the dog was an important clue to solving the mystery.
} 
to high energy (See, e.g., Jones (2000) and references therein). In this case, the maximum obtainable energy is given by $E_{\max }=k e Z u B L$, where $u \leq c$ is the shock speed, $e Z$ is the charge of the particle baing accelerated, $B$ is the magnetic field strength, $L$ is the size of the accelerating region and the numerical parameter $k=\mathcal{O}(1)$ (Drury 1994). Taking $k=1$ and $u=c$, one finds

$$
E_{\text {max }}=0.9 Z(B L)
$$

with $E$ in $\mathrm{EeV}, B$ in $\mu \mathrm{G}$ and $R$ in kpc. This assumes that particles can be accelerated efficiently up until the moment when they can no longer be contained by the source, i.e. until their gyroradius becomes larger than the size of the source. Hillas (1984) used this relation to construct a plot of $B$ vs. $L$ for various candidate astrophysical objects. A "Hillas plot" of this kind, recently constructed by Olinto (2000), is shown in Figure 4.

Given the relationship between $E_{\max }$ and $B L$ as shown in Figure 4, there are not too many astrophysical candidates for zevatrons. Of these, galactic sources such as white dwarfs, neutron stars, pulsars, and magnetars can be ruled out because their galactic distribution would lead to anisotropies above $10 \mathrm{EeV}$ which would be similar to those observed at lower energies by Hayashida et al (1999), and this is not the case. Perhaps the most promising potential zevatrons are radio lobes of strong radio galaxies (Biermann and Strittmatter (1987). The trick is that such sources need to be found close enough to avoid the GZK cutoff (e.g., Elbert and Sommers 1995). Biermann (see these proceedings) has suggested that the nearby radio galaxy M87 may be the source of the observed trans-GZK cosmic rays (see also Stecker 1968; Farrar and Piran 2000). Such an explanation would require one to invoke magnetic field configurations capable of producing a quasi-isotropic distribution of $>10^{20} \mathrm{eV}$ protons, making this hypothesis questionable. However, if the primary particles are nuclei, it is easier to explain a radio galaxy origin for the two highest energy events (Stecker and Salamon 1999; see section VII).

It has also been suggested that since all large galaxies are suspected to harbor super- 
massive black holes in their centers which may have once been quasars, fed by accretion disks which are now used up, that nearby quasar remnants may be the searched-for zevatrons (Boldt and Ghosh 1999; Boldt and Lowenstein 2000). This scenario also has potential theoretical problems and needs to be explored further from a theoretical point-of-view.

Another proposed zevatron, the $\gamma$-ray burst, is discussed in the next section.

\section{GAMMA-RAY BURSTS: AN UNLIKELY SUSPECT}

In 1995, it was suggested that cosmological $\gamma$-ray bursts (GRBs) were the source of the highest energy cosmic rays (Waxman 1995; Vietri 1995). It was suggested that if these objects emitted the same amount of energy in ultrahigh energy $\left(\sim 10^{14} \mathrm{MeV}\right)$ cosmic rays as in $\sim \mathrm{MeV}$ photons, there would be enough energy input of these particles into intergalactic space to account for the observed flux. At that time, it was assumed that the GRBs were distributed uniformly, independent of redshift.

In recent years, X-ray, optical, and radio afterglows of about a dozen GRBs have been detected leading to the subsequent identification of the host galaxies of these objects and consequently, their redshifts. The host galaxies of GRBs appear to be sites of active star formation. The colors and morphological types of the host galaxies are consistent with active star formation as is the detection of Ly $\alpha$ and [OII] in several of these galaxies. Further evidence suggests that bursts themselves are directly associated with star forming regions within their host galaxies; their positions correspond to regions having significant hydrogen column densities with evidence of dust extinction. It now seems more reasonable to assume that a more appropriate redshift distribution to take for GRBs is that of the average star formation rate.

To date, some 14 GRBs afterglows have been detected with a subsequent identification of their host galaxies. At least 13 of the 14 are at moderate to high redshifts with the highest 
one (GRB000131) lying at a redshift of 4.50 (Andersen, et al. 2000).?

A good argument in favor of strong redshift evolution for the frequency of occurrence of the GRBs has been made by Mao and Mo (1998), based on the nature of the host galaxies. Other recent analyses have also favored a GRB redshift distribution which follows the strong redshift evolution of the star formation rate (Schmidt 1999; Fenimore and RamirezRuiz 2000). If we thus assume a redshift distribution for the GRBs which follows the star formation rate, being significantly higher at higher redshifts, GRBs fail by at least an order of magnitude to account for the observed cosmic rays above $100 \mathrm{EeV}$ (Stecker 2000). If one wishes to account for the GRBs above $10 \mathrm{EeV}$, this hypothesis fails by two to three orders of magnitude (Scully and Stecker 2000). Even these numbers are most likely to be too optimistic, since they are based on the questionable assumption of the same amount of GRB energy going into ultrahigh energy cosmic rays as $\sim \mathrm{MeV}$ photons.

Figure 5, from Scully and Stecker (2000), shows the form of the cosmic ray spectrum to be expected from sources with a uniform redshift distribution and sources which follow the star formation rate. The required normalization and spectral index determine the energy requirements of any cosmological sources which are invoked to explain the observations. Pileup effects and GZK cutoffs are evident in the theoretical curves in this figure. As can be seen in Figure 5, the present data appear to be statistically consistent with either the presence or the absence of a pileup effect. Future data with much better statistics are required to determine such a spectral structure.

\footnotetext{
${ }^{2}$ The origin of one burst, GRB980425, is somewhat controversial; a possible X-ray source and an unusual nearby Type Ic supernova have both been put forward as candidates. Taking the supernova identification gives an energy release which is orders of magnitude smaller than that for a typical cosmological GRB.
} 


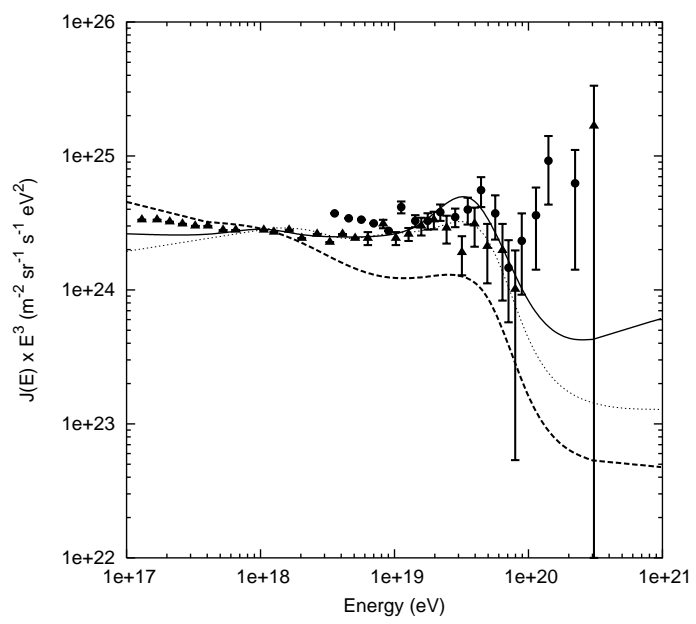

FIG. 5. Predicted spectra for cosmic ray protons as compared with the data. The middle curve and lowest curve assume an $E^{-2.75}$ source spectrum with a uniform source distribution and one that follows the $z$ distribution of the star formation rate respectively. The upper curve is for an $E^{-2.35}$ source spectrum which requires an order of magnitude more energy input and exhibits the "pilup effect" discussed in the text.

\section{HEAVY NUCLEI: A MORE LIKELY SUSPECT}

A more conservative hypothesis for explaining the trans-GZK events is that they were produced by heavy nuclei. Stecker and Salamon (1999) have shown that the energy loss time for nuclei starting out as Fe is longer than that for protons for energies up to a total energy of $300 \mathrm{EeV}$ (see Figure 6).

Stanev et al. (1995) and Biermann (1998) have examined the arrival directions of the highest energy events. They point out that the $\sim 200 \mathrm{EeV}$ event is within $10^{\circ}$ of the direction of the strong radio galaxy NGC 315. NGC 315 lies at a distance of only $\sim 60 \mathrm{Mpc}$ from us. For that distance, the results of Stecker and Salamon (1999) indicate that heavy nuclei 
would have a cutoff energy of $\sim 130 \mathrm{EeV}$, which may be within the uncertainty in the energy determination for this event. The $\sim 300 \mathrm{EeV}$ event is within $12^{\circ}$ of the direction of the

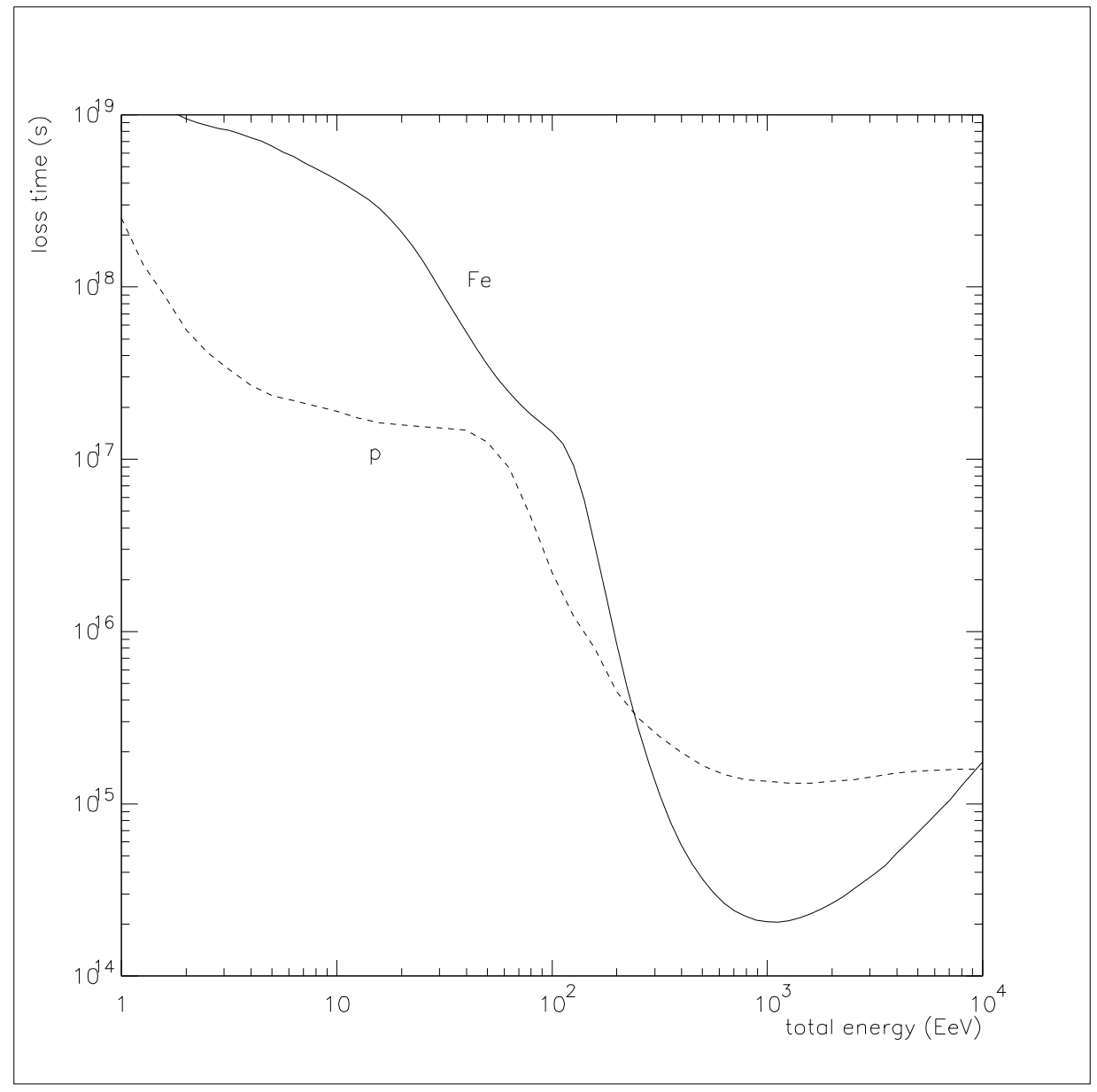

FIG. 6. Mean energy loss times for protons (Stecker 1968; Puget, Stecker and Bredekamp 1976) and nuclei originating as Fe (Stecker and Salamon 1999).

strong radio galaxy $3 \mathrm{C} 134$. The distance to $3 \mathrm{C} 134$ is unfortunately unknown because its location behind a dense molecular cloud in our own galaxy obscures the spectral lines required for a measurement of its redshift. It may be possible that either cosmic ray protons or heavy nuclei originated in these sources and produced the highest energy air shower events. 
An interesting new clue that we may indeed be seeing heavier nuclei above the protonGZK cutoff comes from a very recent analysis of inclined air showers above $10 \mathrm{EeV}$ energy (Ave, et al. 2000). These new results favor proton primaries below the p-GZK cutoff energy but they appear to favor a heavier composition above the $p$-GZK cutoff energy. It will be interesting to see what future data from much more sensitive detectors will tell us.

\section{THE “TOP” SUSPECT (SPILLING GUTS?)}

A way to avoid the problems with finding plausible astrophysical zevatrons is to start at the top, i.e., the energy scale associated with grand unification, supersymmetric grand unification or its string theory equivalent.

The modern scenario for the early history of the big bang takes account of the work of particle theorists to unify the forces of nature in the framework of Grand Unified Theories (GUTs). This concept extends the very successful work of Nobel Laureates Glashow, Weinberg, and Salam in unifying the electromagnetic and weak nuclear forces of nature. As a consequence of this theory, the electromagnetic and weak forces would have been unified at a higher temperature phase in the early history of the universe and then would have been broken into separate forces through the mechanism of spontaneous symmetry breaking caused by vacuum fields, called Higgs fields.

In GUTs, this same paradigm is used to infer that the electroweak force becomes unified with the strong nuclear force at very high energies of $\sim 10^{24} \mathrm{eV}$ which occurred only $\sim$ $10^{-35}$ seconds after the big bang. The forces then became separated owing to interactions with the much heavier mass scale Higgs fields whose symmetry was broken spontaneously. The supersymmetric GUTs (or SUSY GUTs) provide an explanation for the vast difference between the two unification scales (known as the "Hierarchy Problem") and predict that the running coupling constants which describe the strength of the various forces become equal at the SUSY GUT scale of $\sim 10^{24} \mathrm{eV}$.

The fossil remnants of this unification are predicted to be very heavy topological defects 
in the vacuum of space caused by misalignments of the heavy Higgs fields in regions which were causally disconnected in the early history of the universe. These are localized regions where extremely high densities of mass-energy are trapped. Such defects go by designations such as cosmic strings, monopoles, walls, necklaces (strings bounded by monopoles), and textures, depending on their geometrical and topological properties. Inside a topological defect, the vestiges of the early universe may be preserved to the present day. The general scenario for creating topological defects in the early universe was suggested by Kibble (1976).

Superheavy particles or topological structures arising at the GUT energy scale $M \geq 10^{23}$ eV can decay or annihilate to produce "X-particles" (GUT scale Higgs particles, superheavy fermions, or leptoquark bosons of mass M.) In the case of strings this could involve mechanisms such as intersecting and intercommuting string segments and cusp evaporation. These X-particles will decay to produce QCD fragmentation jets at ultrahigh energies, so I will refer to them as "fraggers". QCD fraggers produce mainly pions with a 3 to $10 \%$ admixture of baryons, so that generally one can expect them to produce at least an order of magnitude more ultrahigh energy $\gamma$-rays and neutrinos than protons. The same general scenario would hold for the decay of long-lived superheavy dark matter particles, which will also be fraggers. It has also been suggested that SUSY models which can have an additional soft symmetry breaking scale at TeV energies ("flat SUSY theories") may help explain the observed $\gamma$-ray background flux at energies $\sim 0.1 \mathrm{TeV}$ (Bhattacharjee, Shafi and Stecker 1998).

The number of variations and models for explaining the ultrahigh energy cosmic rays based on the GUT or SUSY GUT scheme (which have come to be called "top-down” models) has grown to be enormous and I will not attempt to list all of the numerous citations involved. Fortunately, Bhattacharjee and Sigl (2000) have recently published an extensive review with over 500 citations and I refer the reader to this review for further details of "top-down" models and references. The important thing to note here is that, if the implications of such models are borne out by future cosmic ray data, they may provide our first real evidence for GUTs! 


\section{A. "Z-bursts"}

It has been suggested that ultra-ultrahigh energy $\mathcal{O}(10 \mathrm{ZeV})$ neutrinos can produce ultrahigh energy $Z^{0}$ fraggers by interactions with $1.9 \mathrm{~K}$ thermal CBR neutrinos (Weiler 1982), resulting in "Z-burst" fragmentation jets, again mostly pions. This will occur at

the resonance energy $E_{\text {res }}=4\left[m_{\nu}(\mathrm{eV})\right]^{-1} \mathrm{ZeV}$. A typical $Z$ boson will decay to produce $\sim 2$ nucleons, $\sim 20 \gamma$-rays and $\sim 50$ neutrinos, $2 / 3$ of which are $\nu_{\mu}$ 's. If the nucleons are produced within a few tens of Mpc they can reach us, even though the original $\sim 10 \mathrm{ZeV}$ neutrinos could have come from a much further distance. The basic problem with this explanation for the trans-GZK events is that one needs to produce $10 \mathrm{ZeV}$ neutrinos. If these are secondaries from pion production, this implies that the primary protons which produce them must have energies of hundreds of ZeV! That is why I have listed this possibility as a sub-section of "top down" models. However, top-down models produce their own fraggers, making Z-bursts a secondary effect.

\section{OTHER NEW PHYSICS POSSIBILITIES}

The GZK cutoff problem has stimulated theorists to look for possible solutions involving new physics. Some of these involve (A) a large increase in the neutrino-nucleon cross section at ultrahigh energies, (B) new particles, and (C) a small violation of Lorentz Invariance (LI).

\section{A. Increasing the Neutrino-Nucleon Cross Section at Ultrahigh Energies}

Since neutrinos can travel through the universe without interacting with the $2.7 \mathrm{~K} \mathrm{CBR}$, it has been suggested that if the neutrino-nucleon cross section were to increase to hadronic values at ultrahigh energies, they could produce the giant air showers and account for the observations of showers above the proton-GZK cutoff. Several suggestions have been made for processes that can enhance the neutrino-nucleon cross section at ultrahigh energies. These suggestions include composite models of neutrinos (Domokos and Nussinov 1987; 
Domokos and Kovesi-Domokos 1988), scalar leptoquark resonance channels (Robinett 1988) and the exchange of dual gluons (Bordes, et al. 1998). Burdman, Halzen and Ghandi (1998) have ruled out a fairly general class of these types of models, including those listed above, by pointing out that in order to increase the neutrino-nucleon cross section to hadronic values at $\sim 10^{20} \mathrm{eV}$ without violating unitarity bounds, the relevant scale of compositeness or particle exchange would have to be of the order of a $\mathrm{GeV}$, and that such a scale is ruled out by accelerator experiments.

However, the interesting possibility exists for a large increase in the number of degrees of freedom above the electroweak scale in models of $\mathrm{TeV}$ scale quantum gravity. It has been suggested that in such models, $\sigma(\nu \mathrm{N}) \simeq\left[E_{\nu} /\left(10^{20} \mathrm{eV}\right)\right] \mathrm{mb}$ (Nussinov and Schrock 1999; Jain, et al. 2000); see also Domokos and Kovesi-Domokos 1999). It should be noted that a cross section of at least $10 \mathrm{mb}$ would be necessary to approach obtaining consistency with the air shower profile data.

\section{B. New Particles}

The suggestion has also been made that new neutral particles containing a light gluino could be producing the trans-GZK events (Farrar 1996; Cheung, Farrar and Kolb 1998). While the invocation of such new particles is an intriguing idea, it seems unlikely that such particles of a few proton masses would be produced in copious enough quantities in astrophysical objects without being detected in terrestrial accelerators. Also there are now strong constraints on gluinos (Alavi-Harati, et al. 1999). One should note that while it is true that the GZK threshold for such particles would be higher than that for protons, such is also the case for the more prosaic heavy nuclei (see section VII). In addition, such neutral particles cannot be accelerated directly, but must be produced as secondary particles, making the energetics reqirements more difficult. 


\section{Breaking Lorentz Invariance}

With the idea of spontaneous symmetry breaking in particle physics came the suggestion that Lorentz invariance (LI) might be weakly broken at high energies (Sato and Tati 1972). Although no real quantum theory of gravity exists, it was suggested that LI might be broken as a consequence of such a theory (Amelino-Camilia et al. 1998). A simpler formulation for breaking LI by a small first order perturbation in the electromagnetic Lagrangian which

leads to a renormalizable treatment has been given by Coleman and Glashow (1999). Using this formalism, these authors have shown than only a very tiny amount of LI symmetry breaking is required to avoid the GZK effect by supressing photomeson interactions between ultrahigh energy protons and the CBR. Of course, this would also eliminate any "pileup" structure below the predicted GZK cutoff energy.

\section{SMOKING GUNS}

Future data which will be obtained with new detector arrays and satellites (see next section) will give us more clues relating to the origin of the trans-GZK events by distinguishing between the various hypotheses which have been proposed.

A zevatron origin ("bottom-up" scenario) will produce air-showers primarily from primaries which are protons or heavier nuclei, with a much smaller number of neutrino induced showers. The neutrinos will be secondaries from the photomeson interactions which produce the GZK effect (Stecker 1973; 1979; Hill and Schramm 1985; Stecker et al 1991). In addition, zevatron events may cluster near the direction of the sources.

A "top-down" (GUT) origin mechanism will not produce any heavier nuclei and will produce at least an order of magnitude more ultrahigh energy neutrinos than protons. Thus, it will be important to look for the neutrino-induced air showers which are expected to originate much more deeply in the atmosphere than proton-induced air showers and are therefore expected to be mostly horizontal showers. Looking for these events can most 
easily be done with a satellite array which scans the atmosphere from above (see next section). The "top down" model also produces a large ratio of ultrahigh energy photons to protons, however, the mean free path of these photons against pair-production interactions with extragalactic low frquency radio photons from radio galaxies is only a few Mpc at most (Protheroe and Biermann 1996). The subsequent electromagnetic cascade and synchrotoron emission of the high energy electrons produced in the cascade dumps the energy of these particles into much lower energy photons (Wdowczyk, Tkaczyk and Wolfendale 1972; Stecker 1973).

Another distinguishing characteristic between bottom-up and top-down models is that the latter will produce much harder spectra. If differential cosmic ray spectra are parametrized to be of the form $F \propto E^{-\Gamma}$, then for top-down models $\Gamma<2$, whereas for bottom-up models $\Gamma \geq 2$. Also, because of the hard source spectrum in the "top-down" models, they should exhibit both a GZK suppression and a pileup just before the GZK energy.

If Lorentz invariance breaking is the explanation for the missing GZK effect, the actual absence of photomeson interactions should result the absence of a pileup effect as well.

\section{THE NEW DETECTIVES}

Of the ground-based ultra-high energy arrays, the AGASA array of particle detectors in Japan continues to get ultrahigh energy cosmic ray data. Its aperture is $200 \mathrm{~km}^{2} \mathrm{sr}$.

The HiRes array is operating and will soon be publishing data. This array is an extension of the Fly's Eye which pioneered the technique of measuring the atmospheric fluorescence light in the near UV (300 - $400 \mathrm{~nm}$ range) that is isotropically emitted by nitrogen molecules that are excited by the charged shower secondaries at the rate of $\sim 4$ photons per meter per particle. Its estimated aperture is $1000 \mathrm{~km}^{2} \mathrm{sr}$ at $100 \mathrm{EeV}$ with a $10 \%$ duty cycle (Sokolsky 1998) and it has already detected several events above the GZK cutoff energy (Abu-Zayyad, PhD thesis 2000). 
The southern hemisphere Auger array is expected to be on line in the near future. This will be a hybrid array which will consist of 1600 particle detector elements similar to those at Havera Park and three or four flourescence detectors. Its expected aperture will be 7000 $\mathrm{km}^{2} \mathrm{sr}$ for the ground array above $10 \mathrm{EeV}$ and $\sim 10 \%$ of this number for the hybrid array.

The Telescope Array will will consist of eight separate flourescence detecting telescope stations separated by $30 \mathrm{~km}$. Its expected aperture will be $8000 \mathrm{~km}^{2} \mathrm{sr}$ with an assumed $10 \%$ duty cycle.

The next big leap will be to go to a system of space-based detectors which will look down on the Earth's atmosphere to detect the trails of nitrogen flourescence light made by giant extensive air showers. The Orbiting Wide-angle Light collectors (OWL) mission is being proposed to study such showers from satellite-based platforms in low Earth orbit (600 - $1200 \mathrm{~km}$ ). OWL would observe extended air showers from space via the air fluorescence technique, thus determining the composition, energy, and arrival angle distributions of the primary particles in order to deduce their origin. Operating from space with a wide fieldof-view instrument dramatically increases the observed target volume, and consequently the detected air shower event rate, in comparison to ground based experiments. The OWL baseline configuration will yield event rates that are more than two orders of magnitude larger than currently operating ground-based experiments. The estimated aperture for a two satellite system is $3 \times 10^{5} \mathrm{~km}^{2} \mathrm{sr}$ above a few tens of EeV assuming a $10 \%$ duty cycle.

Figure 7 illustrates two OWL satellites obtaining stereoscopic views of an air shower produced by an ultra-high energy cosmic ray. With an approximate $10 \%$ duty factor, OWL will be capable of making accurate measurements of giant air shower events with high statistics. It is expected to be able to detect more than 1000 showers per year with $E \geq$ $100 \mathrm{EeV}$ (assuming an extrapolation of the cosmic ray spectrum based upon ground-based measurements).

Closer in the future, the European Space Agency is studying the feasibility of placing such a light collecting detector on the International Space Station in order to develop the required technology to observe the flourescent trails of giant extensive air showers, to make 
such observations, and to serve as a pathfinder mission for a later free flyer. This experiment has been dubbed the Extreme Universe Space Observatory (EUSO) (see paper of Livio Scarsi, these proceedings, for more details). Owing to the orbit parameters and constraints of the International Space Station, the effective aperture for EUSO will not be as large as that of a free flyer mission.

A recent compendium of papers on observing giant air showers from space may be found in Krizmanic, Ormes and Streitmatter (1998).

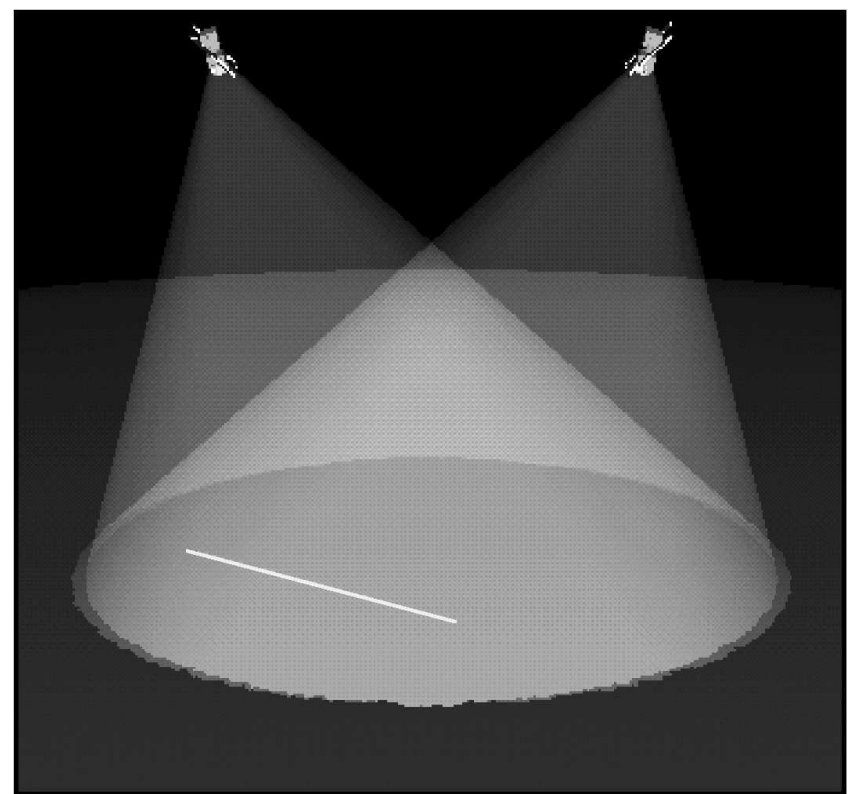

FIG. 7. Two OWL satellites in low-Earth orbit observing the flourescent track of a giant air shower. The shaded cones illustrate the field-of-view for each satellite.

\section{THE MYSTERY REMAINS}

With regard to the ultrahigh energy cosmic rays, we are, as Sherlock Holmes would have said, "suffering from a plethora of surmise, conjecture, and hypothesis". Indeed, since "The Adventure of Silver Blaze", from which I have now twice quoted, concerns a race horse, it seems appropriate language to say that many high energy theorists, given free rein, have grabbed the bit between their teeth and bolted off to far pastures.

It seems fair to say that every solution which I have discussed in these lectures, and more particularly, those which I have chosen not to discuss, suffer from obvious problems 
or potential problems. Lets us then recall that we could not understand how the sun shines until we had an understanding of nuclear reactions. Perhaps future cosmic ray investigations will also lead to one of those unexpected solutions in physics and astrophysics which will result in truly new knowledge.

\section{ACKNOWLEDGMENTS}

I would like to thank Tom Gaisser, Angela Olinto and Alan Watson for sending me files of their figures and for allowing me to use them in this paper. I would also like to thank John Krizmanic for his help with section XI and for supplying Figure 7. 


\section{REFERENCES}

Abu-Zayyad, T. et al. 2000, Phys. Rev. Letters 84, 4276

Alavi-Harati, A.et al. 1999, Phys. Rev. Letters 83, 2128

Amilino-Camilia, G. et al. 1998, Nature 393, 763

Ave, M. et al. 2000, Phys. Rev. Letters 852244

Berezinsky, V.S. and Grigor'eva S.I. 1988, Astronomy and Astrophys. 199, 1

Biermann, P.L. 1998, in Workshop on Observing Giant Cosmic Ray Air Showers from Space

ed. J.F. Krizmanic, J.F. Ormes and R.E. Streitmatter (New York: American Institute of Physics), p.22

Bhattacharjee, P., Shafi, Q. and Stecker, F.W. 1998, Phys. Rev. Letters 80, 3698

Bhattacharjee, P. and Sigl 2000, Physics Reports 327, 109

Bird, D.J. et al. 1993, Phys. Rev. Letters 71, 3401

Bird, D.J. et al. 1994, Astrophys. J. 424, 491

Boldt, E. and Ghosh, P. 1999, Monthly Notices Royal Astr. Soc. 307, 491

Boldt, E. and Lowenstein, M. 2000, Monthly Notices Royal Astr. Soc. 316, L29

Bordes, J. et al. 1998, Astroparticle Physics 8, 135

Burdman, G., Halzen, F. and Ghandi, R. 1998, Physics Letters B417, 107

Cheung, D.J.H., Farrar, G.R. and Kolb, E.W. 1998,Physical Review D57, 4606

Coleman, S. and Glashow, S.L. 1999, Phys. Rev. D59, 116008

Domokos, G. and Kovesi-Domokos, S. 1988 Phys. Rev. D38, 2833

Domokos, G. and Kovesi-Domokos, S. 1999, Phys. Rev. Letters 82, 1366 
Domokos, G. and Nussinov, S. 1987, Physics Letters B187, 372

Drury, L. 1994, Contemporary Physics 35, 232

Elbert, J.W. and Sommers, P. 1995, Astrophys. J. 441, 151

Farrar, G.R. 1996, Phys. Rev. Letters 76, 4111

Farrar, G.R. and Piran, T. 2000, Phys. Rev. Letters 84, 3527

Fenimore, E.E. and Ramirez-Ruiz 2000, e-print astro-ph/0004176

Gaisser, T.K. 2000, e-print astro-ph/0011525

Greisen, K. 1966, Phys. Rev. Letters 16, 748

Hayashida, N., et al. 1999, Astroparticle Phys. 10, 303

Hill, C.T. and Schramm, D.N. 1985, Phys. Rev. D31, 564

Hillas, A.M. 1984. Annual Review of Astronomy and Astrophysics 22, 425

Jain, P., et al. 2000, Physics Letters B484, 267

Jones, T.W. 2000, e-print astro-ph/0012483

Kibble, T.W.B. 1976, Journal of Physics A 9, 1387

Krizmanic, J.F. Ormes, J.F. and Streitmatter, R.E. 1998, ed. Workshop on Observing Giant Cosmic Ray Air Showers from Space, (New York: American Institute of Physics)

Linsley, J. 1963, Phys. Rev. Letters 10, 146

Mao, S. and Mo, H.J. 1998, Astronomy and Astrophys. 339, L1

Mather, J. et al. 1994, Astrophys. J. 420, 439

Nagano, M. and Watson, A.A. 2000, Reviews of Modern Physics72, 689

Nussinov, S. and Schrock, R. 1999, Phys. Rev. D59, 105002 
Olinto, A. 2000, e-print astro-ph/0011106

Protheroe, R.J. and Biermann, P.L. 1996, Astroparticle Phys. 6, 45

Puget, J.L., Stecker, F.W. and Bredekamp, J. 1976, Astrophys. J. 205, 638

Penzias, A.A. and Wilson, R.W. 1965, Astrophys. J. 142, 419

Robinett, R.W. 1988, Phys. Rev. D37, 84

Sato, H. and Tati, T. 1972, Progress in Theoretical Physics 47, 1788

Schmidt, M. 1999, Astrophys. J. 523, L117

Scully, S.T. and Stecker, F.W. 2000, Phys. Rev. Letters, submitted, e-print astroph/0006112

Smoot, G. et al. 1992, Astrophys. J. 396, L1

Sokolsky, P. 1998, in Workshop on Observing Giant Cosmic Ray Air Showers from Space ed. J.F. Krizmanic, J.F. Ormes and R.E. Streitmatter (New York: American Institute of Physics), p. 65

Stanev, T. et al. 1995, Phys. Rev. Letters 75, 3056

Stecker, F.W. 1968, Phys. Rev. Letters 21, 1016

Stecker, F.W. 1973, Astrophys. and Space Sci. 20, 47

Stecker, F.W. 1979, Astrophys. J. 228, 919

Stecker, F.W. 1989, Nature 342, 401

Stecker, F.W. 2000, Astroparticle Phys. 14, 207

Stecker, F.W. and Salamon, M.H. 1999, Astrophys. J. 512, 521

Stecker, F.W. et al. 1991, Phys. Rev. Letters 66, 2697 
Takeda, M. et al. 1998, Phys. Rev. Letters 81, 1163

Takeda, M. et al. 1999, Astrophys. J. 522, 225

Vietri, M. 1995, Astrophys. J. 453, 883

Waxman, E. 1995, Phys. Rev. Letters 75, 386

Wdowczyk, J., Tkaczyk, T. and Wolfendale, A.W. 1972 J. Phys. A5, 1419

Weiler, T.J. 1982, Phys. Rev. Letters 49, 234

Zatsepin, G.T. and Kuz'min, V.A. 1966, Zh.Eksp.Teor.Fiz., Pis'ma Red.4, 144 